\title{
Role of Wnt5a in periodontal tissue development, maintenance, and periodontitis: Implications for periodontal regeneration (Review)
}

\author{
XIUQUN WEI ${ }^{1-3}$, QIAN LIU ${ }^{1-3}$, SHUJUAN GUO ${ }^{1-3}$ and YAFEI WU ${ }^{1,3}$ \\ ${ }^{1}$ State Key Laboratory of Oral Diseases, National Clinical Research Center for Oral Diseases; \\ ${ }^{2}$ National Engineering Laboratory for Oral Regenerative Medicine; ${ }^{3}$ Department of Periodontics, \\ West China Hospital of Stomatology, Sichuan University, Chengdu, Sichuan 610041, P.R. China
}

Received September 2, 2020; Accepted November 25, 2020

DOI: $10.3892 / \mathrm{mmr} .2020 .11806$

\begin{abstract}
The periodontium is a highly dynamic microenvironment constantly adapting to changing external conditions. In the processes of periodontal tissue formation and remodeling, certain molecules may serve an essential role in maintaining periodontal homeostasis. Wnt family member 5a (Wnt5a), as a member of the Wnt family, has been identified to have extensive biological roles in development and disease, predominantly through the non-canonical Wnt signaling pathway or through interplay with the canonical Wnt signaling pathway. An increasing number of studies has also demonstrated that it serves crucial roles in periodontal tissues. Wnt5a participates in the development of periodontal tissues, maintains a non-mineralized state of periodontal ligament, and regulates bone homeostasis. In addition, Wnt5a is involved in the pathogenesis of periodontitis. Recently, it has been shown to serve a positive role in the regeneration of integrated periodontal complex. The present review article focuses on recent research studies of Wnt5a and its functions in development, maintenance, and pathological disorders of periodontal tissues, as well as its potential effect on periodontal regeneration.
\end{abstract}

\section{Contents}

1. Introduction

2. Wnt5a signaling and functions

3. Wnt5a in periodontal development

4. Wnt5a in periodontal tissue maintenance

Correspondence to: Professor Yafei Wu or Dr Shujuan Guo, Department of Periodontics, West China Hospital of Stomatology, Sichuan University, 14 Renmin South Road (3rd Sect), Chengdu, Sichuan 610041, P.R. China

E-mail: yfw1110@163.com

E-mail: guo.shujuan@yahoo.com

Key words: Wnt family member 5a, periodontium, periodontal homeostasis, periodontitis, periodontal regeneration
5. Wnt5a in periodontitis

6. Wnt5a in periodontal regeneration

7. Conclusion

\section{Introduction}

Periodontal tissues, mainly derived from the dental follicle (DF), comprise the gingiva, cementum, alveolar bone, and periodontal ligament (PDL) (1). The periodontium functions as a unit to support and invest the tooth, disperse occlusal force, and maintain the long-term stability of dentognathic system. The periodontium is a highly dynamic microenvironment that constantly undergoes turnover; under normal conditions, the rates of tissue formation are in balance with the rates of tissue degradation (2). External stimuli, such as microorganisms, mechanical stress, or any factors that may cause developmental defects or immune disorders, can affect this dynamic balance (3-5). Subsequently, the host can raise responses to these challenges through various factors and molecules, in order to achieve a new dynamic balance $(6,7)$.

The Wnt signaling pathway has long been considered to serve an essential role in the development and disease of periodontal tissues. Canonical Wnt signaling (through $\beta$-catenin) was recently confirmed to be associated with the development of cementum and constitutive activation of $\beta$-catenin might cause cementum hyperplasia (8). Wnt family member $5 \mathrm{a}$ (Wnt5a), a prototypical $\beta$-catenin-independent Wnt family member, has also been found to be highly involved in periodontal tissue development and periodontitis.

Wnt5a may participate in the development of periodontal tissues, maintaining a non-mineralized state of periodontal ligament, and regulating bone homeostasis. In periodontitis, excessive production of Wnt5a might function as a proinflammatory factor amplifying local inflammation. However, other studies have reported a positive role for Wnt5a in the regeneration of integrated periodontal complex. The present review article focused on the current understanding of Wnt5a and its functions in periodontal tissue development, maintenance, and periodontitis, as well as its potential effect on periodontal regeneration. 


\section{Wnt5a signaling and functions}

The Wnt5a cDNA was first isolated from fetal mice (9) and was later sequenced in humans (10). The Wnt5a gene, which is located on chromosome 3p14-p21, has been identified to encode two different protein isoforms, Wnt5a-long (Wnt5a-L) and Wnt5a-short (Wnt5a-S), due to alternative transcriptional start sites. The protein Wnt5a-L is 380 amino acids in size, and Wnt5a-S comprises 365 amino acids (11). Biochemical analysis has demonstrated that these two purified proteins are similar in stability, hydrophobicity, and Wnt/ $\beta$-catenin signaling activity. However, they are differentially expressed in tissues and might serve distinct roles in cancer. It has been reported that Wnt5a-L inhibits, while Wnt5a-S promotes, the growth of tumor cell lines (11).

The receptors of Wnt5a mainly include receptor tyrosine kinase-like orphan receptors (RoRs), the frizzled family of receptors (Fzd receptors), and co-receptors low-density lipoprotein receptor-related protein (Lrp) 5 and 6 . When binding to these receptors, Wnt5a exerts its intracellular effects predominantly by activating two non-canonical Wnt signaling pathways (Fig. 1) (12). One branch is the planar cell polarity pathway, which involves the activation of small GTPases Rho and Rac, and the subsequent regulation of c-Jun $\mathrm{N}$-terminal kinase (Jnk) to control cell orientation (13). The other branch is the calcium pathway, which when activated, increases the intracellular $\mathrm{Ca}^{2+}$ levels, subsequently activating calcium/calmodulin dependent protein kinase II (CaMKII) and protein kinase $\mathrm{C}$. This signaling pathway has been demonstrated to mediate cell proliferation, migration, and adhesion (14). Notably, in addition to activating the non-canonical Wnt pathway, Wnt5a can have regulatory effects on the canonical Wnt/ $\beta$-catenin signaling pathway to control cell proliferation and differentiation (Fig. 1), depending on the receptor and cell type context (15). Secreted frizzled-related protein 5 (Sfrp5), a soluble protein, is one of the most common Wnt5a antagonists. Sfrp5 has been demonstrated to inhibit Wnt5a signaling in both physiological and pathological processes (16).

Wnt5a has been shown to be highly conserved among species and associated with many processes of development and disease. Homozygous Wnt5a-knockout mouse embryos are perinatally lethal (17). In addition, Wnt5a affects the morphogenesis of internal organs, such as the heart (18) and the lungs (19). It is associated with proinflammatory factor production and immune cell recruitment in inflammation (20), and related to the occurrence and prognosis of breast and colorectal cancers (21). Of note, previous studies have reported that Wnt5 is involved in tooth growth, patterning, odontoblast differentiation (22) and determining tooth size (23).

\section{Wnt5a in periodontal tissue development}

Wnt5a is expressed at all stages of periodontal tissue development. An early study demonstrated that Wnt5a mRNA was mainly expressed in the dental follicle in developing murine tooth germ (24), while later studies reported that Wnt5a was strongly expressed in both the dental epithelium and mesenchyme $(23,25)$. In postnatal periodontium, Wnt5a expression is robust in the alveolar bone, as well as in the ameloblast and odontoblast layers (26). Wnt5a can also be expressed in mature periodontal tissues and is closely related to the maintenance of periodontal soft and hard tissues under physiological conditions $(27,28)$. Hasegawa et al (27) found that Wnt5a was predominantly detected in mature PDL in rats. Similarly, Wnt5a and its receptors (Ror2, Fzd2, Fzd4 and Fzd5) were expressed in human periodontal ligament cells (HPDLCs) (27).

The expression of Wnt5a in the periodontium appears to be mediated by physiological mechanical stress of occlusion and mastication. Mechanical stress caused by stretch loading augmented the expression levels of Wnt5a and its receptors in HPDLCs, while the removal of occlusal pressure decreased Wnt5a expression levels in rat PDL tissue (27). Wnt5a was highly expressed at the tension sites of PDL and might further contribute to osteogenesis through activation of canonical Wnt signaling through its receptors Fzd4 and Lrp5 in an orthodontic tooth movement model (28).

Wnt5a is hypothesized to participate in root formation and tooth eruption, and further affect the development and formation of cementum and alveolar bone (1). It was observed that the expression levels of Wnt5a increased gradually from the center of the dental follicle (beneath the apical foramen) to the lateral coronal corner, where the DF differentiates into the periodontium during tooth root development in swine (29). This spatial gradient expression suggested the possible regulatory effect of Wnt5a on the differentiation of DF. It is consistent with an earlier study reporting that Wnt5a was expressed on precementoblasts/cementoblasts during late tooth root development (30). Furthermore, Wnt5a is closely related to the growth and modeling of alveolar bone in the process of tooth eruption. The expression of Wnt5a in rat alveolar bone was increased during tooth eruption (31), especially during the osteoclastogenesis burst (3-11 days postnatal) (26). Therefore, the spatial distribution regulation of Wnt5a in alveolar bone may be crucial because tooth eruption depends on the coordination of osteogenesis and osteoclastogenesis in time and space.

\section{Wnt5a in periodontal tissue maintenance}

Despite containing stem cells that retain the potential to differentiate into osteoblasts, cementoblasts and fibroblasts (1), the mature periodontal ligament never ossifies under physiological conditions, a phenomenon known as the periodontal mineral homeostasis. The Wnt pathway has been identified to be involved in the process, and especially the Wnt antagonist Sfrp1. It has been demonstrated that selective inhibition of Sfrpl increased PDLC mineralization and expression levels of mineralization-related genes, including $\beta$-catenin, alkaline phosphatase, osteocalcin, collagen I, and runt-related transcription factor 2 (32). Hasegawa et al (27) found that Wnt5a suppressed mineralization but enhanced collagen production in HPDLCs. In specific, Wnt5a upregulated the expression of periostin through transforming growth factor- $\beta 1$ (TGF $\beta 1$ ) (27), and periostin is essential for the integrity and function of the periodontal ligament $(33,34)$. These findings suggest that Wnt5a may be a positive regulator in fibrillogenesis to remodel PDL tissue and accelerate maturation of PDL fibers, and to prevent non-physiological mineralization (Fig. 2). 


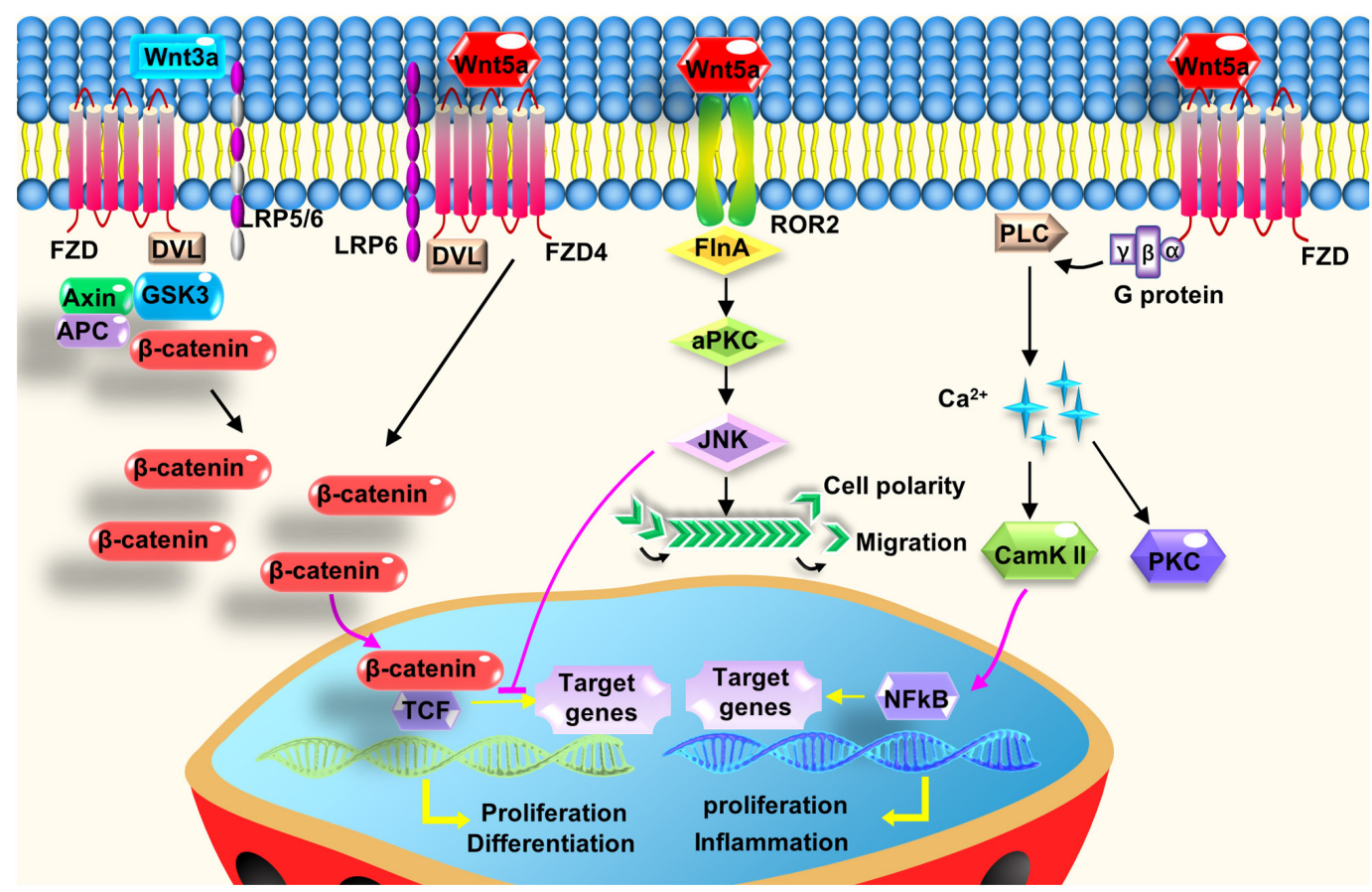

Figure 1. Wnt5a exerts its intracellular effects by activating two non-canonical Wnt signaling pathways, or the interplay with the canonical Wnt signaling pathway. Wnt5a regulates cell polarity, proliferation, migration, and adhesion, predominantly through its downstream planar cell polarity and calcium pathway. Wnt5a can antagonize canonical Wnt signaling mostly by inhibiting the downstream part of $\beta$-catenin/Tcf-mediated transcription or other mechanisms. In the presence of FZD4 and LRP5, Wnt5a stabilizes $\beta$-catenin to activate the canonical Wnt signaling pathway. APC, adenomatous polyposis coli; CaMKII, calcium/ calmodulin dependent protein kinase II; DVL, disheveled; FlnA, filamin A; FZD, frizzled; GSK3, glycogen synthase kinase 3; LRP, low-density lipoprotein receptor-related protein; PKC, protein kinase C; PLC, phospholipase C; Tcf, T-cell factor; Wnt5a, Wnt family member 5a.

In addition to the stability of soft tissues, the maintenance of hard tissues in periodontium is equally important. An early study showed that Wnt5a overexpression mediated dental follicle cells (DFCs) to undergo moderate osteogenesis by augmenting receptor activator of nuclear factor- $\mathrm{KB}$ (RANK) ligand expression but not osteoprotegerin (26). This suggested that Wnt5a might participate in bone homeostasis of periodontal tissues. Several later studies confirmed the role of Wnt5a in regulating bone homeostasis. Wnt5a has been reported to promote osteoclast fusion in bone marrow-derived macrophages by antagonizing another non-canonical Wnt signaling molecule, Wnt16 (35). Osteoblast-derived Wnt5a, when binding to Ror2, increased RANK expression in osteoclast precursor cells through the Jnk pathway, thereby promoting osteoclast differentiation and bone resorption (36). Conversely, conditional deletion of Wnt5a in mature osteoclasts resulted in reduced bone mass by decreasing bone formation with unchanged osteoclast numbers (37). It is generally hypothesized that the nature of the response to Wnt5a is determined to a large extent by the responding cells, and it might contribute to the bone homeostasis rather than promoting osteogenesis or osteoclastogenesis unilaterally.

\section{Wnt5a in periodontitis}

Wnt5a is associated with periodontitis. A previous genome-wide association study has demonstrated that the Wnt5a gene is correlated with severe periodontitis (38). Wnt5a mRNA expression levels in gingival tissues were upregulated in patients with chronic periodontitis compared with healthy subjects (39-41). In addition, the expression levels of Wnt5a mRNA increased in gingival tissues from patients with aggressive periodontitis compared those with chronic periodontitis (41). Furthermore, a positive association was identified between the mRNA expression levels of Wnt5a and the clinical parameters of periodontitis, including clinical attachment loss (CAL) and periodontal pocket depth (PPD) (41). A cross-sectional study revealed that severely diseased sites from patients with generalized forms of moderate and severe chronic periodontitis ( $\geq 30 \%$ of the sites with PPD $\geq 4 \mathrm{~mm}$, CAL $\geq 3 \mathrm{~mm}$ and presence of bleeding on probing) exhibited statistically significantly higher levels of Wnt5a in gingival crevicular fluid (GCF) compared with healthy sites within the same patient (42).

Wnt5a regulates the innate immune response to periodontal pathogen invasion. It is generally accepted that the interplay between subgingival plaque biofilm and host immune response has an important role in the occurrence and development of periodontitis. Previous studies have indicated that Wnt5a is highly involved in the innate immune response of periodontitis, in which gingival epithelial cells and monocyte/macrophage-lineage cells act as barriers to prevent pathogen invasion. Maekawa et al (40) found that Wnt5a was predominantly localized in the epithelial layer, rather than in the subjacent connective tissue, and that Wnt5a expression in human gingival epithelial cells (HGECs) was significantly upregulated following a 12-h stimulation with Porphyromonas gingivalis-derived (Pg-) lipopolysaccharide (LPS) (0.1 and $1.0 \mu \mathrm{g} / \mathrm{ml}$ ) (40). Another study demonstrated that monocytes, but not human gingival fibroblasts (HGFs), had an important role in upregulating Wnt5a expression at inflamed gingiva 


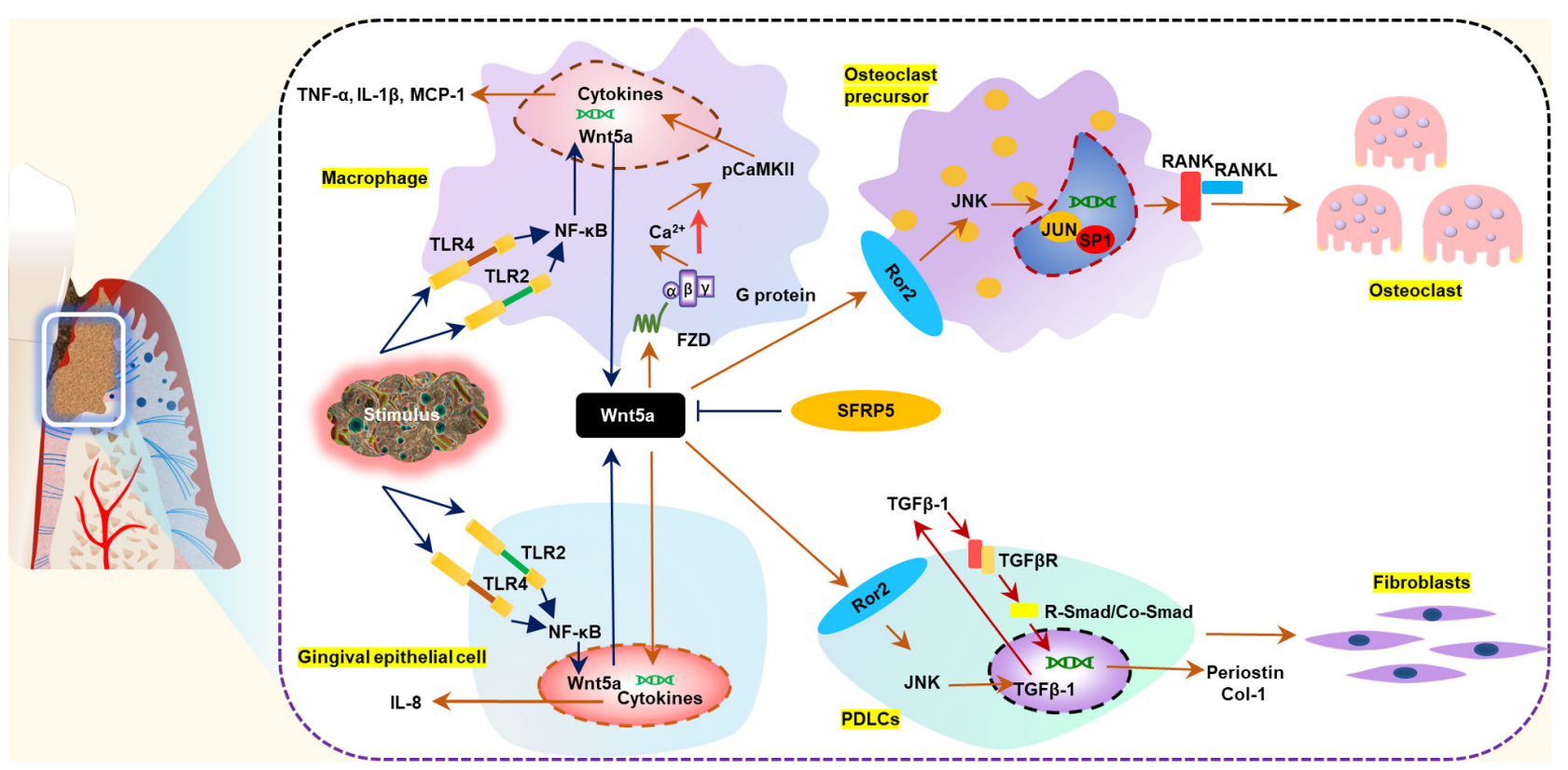

Figure 2. Wnt5a participates in the pathogenesis of periodontitis and regulates periodontal non-mineralized homeostasis and bone homeostasis. Under physiological conditions, Wnt5a contributes to maintaining a non-mineralized state of periodontal ligament and regulating bone homeostasis by promoting fibrillogenesis of periodontal ligament cells and osteoclastogenesis of osteoclast precursors. During the occurrence and development of periodontitis, Wnt5a expression levels increase in response to pathogen stimulation. Excessive production of Wnt5a amplifies the inflammatory response and accelerates the destruction of periodontal tissues. Sfrp5 antagonizes the proinflammatory effects of Wnt5a. CaMKII, calcium/calmodulin dependent protein kinase II; Col-1, collagen 1; FZD, frizzled;

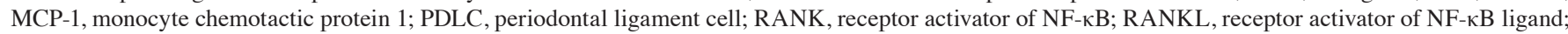
Ror2, receptor tyrosine kinase-like orphan receptor 2; Sfrp5, secreted frizzled-related protein 5; TLR, toll-like receptor; Wnt5a, Wnt family member 5a.

sites (39). The expression levels of Wnt5a mRNA were constant following treatment with $1 \mu \mathrm{g} / \mathrm{ml}$ Pg-LPS in HGFs, while they were significantly increased at $2 \mathrm{~h}$ and reached peak value at $4 \mathrm{~h}$ following Pg-LPS stimulation in primary monocytes (39). Additionally, silencing of toll-like receptor (Tlr)2 and Tlr4 by RNA interference downregulated the Pg-LPS-induced Wnt5a expression (39). Luciferase reporter assay and electrophoretic mobility analysis confirmed that this process was dependent on the NF- $\kappa \mathrm{B}$ signaling pathway, and the addition of $\mathrm{NF}-\kappa \mathrm{B}$ inhibitors (wedelolactone or the inhibitor of $\kappa \mathrm{B}$ kinase inhibitor VII) could reduce the expression of Wnt5a in a dose-dependent manner (39). Further studies indicated that p65, one of the classical $\mathrm{NF}-\kappa \mathrm{B}$ transcription factors, may upregulate Wnt5a via inflammatory stimulation $(43,44)$. Finally, Pg-LPS and interferon- $\gamma$ synergistical stimulation activated Wnt5a in monocytes, partly through the glycoprotein130/Janus kinase/STAT1 signaling pathway (39).

Wnt5a triggers proinflammatory signaling cascades and increases secretion of proinflammatory cytokines. Wnt5a has been demonstrated to regulate inflammatory cytokines and to aggravate the destruction of periodontium. Inhibition of Wnt5a by small interfering (si)RNA or a neutralizing antibody significantly reduced the expression of interleukin (IL)-1 $\beta$, monocyte chemotactic protein 1 , and matrix metalloproteinase 2 in $P$. gingivalis-infected macrophages (45). Local administration of exogenous Wnt5a in the gingiva of mice with ligature-induced periodontitis exacerbated alveolar bone loss (40). It has been reported that the calcium pathway mediated by the Wnt5a receptor Fzd5 might be involved in the process, since Wnt5a stimulation increased intracellular calcium levels and activated CaMKII (46).
The effects of Wnt5a on periodontitis have also been found to be linked to the interaction with its antagonist Sfrp5 (47). It was demonstrated that Sfrp5 may have a protective role in periodontitis to prevent LPS-induced inflammation, whereas Wnt5a was proinflammatory. The expression of Sfrp5 in gingival tissues from patients with periodontitis was significantly decreased, which was consistent with in vitro results from HGECs following Pg-LPS stimulation. Additionally, Sfrp5 inhibited the increase of IL- 8 mRNA expression mediated by Pg-LPS and/or Wnt5a stimulation. Local treatment of Sfrp5 in mice with ligature-induced periodontitis significantly reduced the expression of Wnt5a as well as that of proinflammatory factors (IL-1 $\beta$, IL-6, and IL-17), and inhibited alveolar bone loss (40). In addition, the Sfrp5/Wnt5a axis might have a role in the association between periodontitis and systemic inflammatory disorders (including obesity, type 2 diabetes, and coronary artery disease). A nested case-control study found that in overweight individuals (body mass index, 24-38 kg/m²), Sfrp5 serum levels in patients with periodontitis involving tooth loss were significantly lower than in patients with periodontitis without tooth loss and the matched control group, while Wnt5a had no significant difference (48). Obese human subjects had significantly lower serum Sfrp5 levels than lean controls, while they had significantly higher serum Wnt5a levels (49). Similarly, this inverse pattern of expression for Sfrp5 and Wnt5a was detected in the serum of patients with coronary artery disease (50) and the plasma of patients with type 2 diabetes mellitus (51). An in vitro study confirmed that Sfrp5 alleviated the endothelial dysfunction damage mediated by Wnt5a (52).

Taken together, Wnt5a may serve an important role in periodontitis. It not only regulates the innate immune response to periodontal pathogen invasion, but also triggers 
proinflammatory signaling cascades and increases secretion of proinflammatory cytokines, ultimately resulting to the destruction of periodontium (Fig. 2). However, Wnt5a has been reported to have an anti-inflammatory role under certain conditions. It might be crucial to the functions of macrophages in maintaining CD14 expression, an important immune response mediator, and supporting macrophage survival (44). Wnt5a induced the expression of anti-inflammatory mediator IL-10 in primary human monocytes (53), and promoted macrophage M2 polarization in kidney fibrosis (54). In addition, Wnt5a was found to have an important role in killing Mycobacterium tuberculosis by enhancing autophagy of human monocyte-derived macrophages (55). Thus, the specific role of Wnt5a in inflammation remains inconclusive, and it may depend on the tissue-specific immune microenvironment or the stage of immune response. Further studies are required to fully comprehend the exact role of Wnt5a in periodontitis.

\section{Wnt5a in periodontal regeneration}

Untreated periodontitis leads to periodontal tissue destruction and tooth loss. In recent years, periodontal tissue engineering has been greatly developed, and stem cell therapy remains a research hotspot (56). Previous studies have revealed that the canonical Wnt signaling pathway is essential in periodontal tissue regeneration, especially in stem cell self-renewal and multi-directional differentiation $(57,58)$. The non-canonical Wnt signaling pathways have also been observed to regulate the biological behavior of periodontal stem cells.

Wnt5a may regulate the biological behavior of periodontal stem cells. Wnt5a inhibited the induction of DFCs cellular senescence, supported cell proliferation and prevented cell death (59). Wnt5a significantly enhanced the proliferation of HPDLCs by phosphorylating two major mitogen-activated protein kinases, extracellular signal-regulated kinase and Jnk (27), which are important signaling molecules influencing cell proliferation (60). In addition, Wnt5a promoted the migration of HPDLCs by phosphorylating Akt, which serves pivotal roles in cell migration (61). Similar to its function in bone homeostasis, Wnt5a has dual effects on osteogenic differentiation of periodontal stem cells. Wnt5a suppressed osteoblastic differentiation via Ror2/Jnk signaling in HPDLCs (62). Additionally, Sakisaka et al (30) found that Wnt5a might function as a negative regulator in the Wnt3a-mediated osteogenic differentiation of DFCs by inhibiting the downstream part of $\beta$-catenin/T-cell factor-mediated transcription. As a canonical Wnt family member, Wnt3a has been reported to have a potential role in stimulating cementoblast/osteoblast differentiation of dental follicle cells via the Wnt/ $\beta$-catenin signaling pathway (63). Several studies have suggested that Wnt5a was a positive regulator in osteogenic differentiation of stem/progenitor cells. Xiang et al (26) found that in the presence of $100 \mathrm{ng} / \mathrm{ml}$ bone morphogenetic protein (BMP)2, Wnt5a strongly prompted mineralization of DFCs. Another study indicated that Wnt5a signaling might be a substantial constituent in BMP2-mediated osteoblastogenesis (64). In addition, Wnt5a-induced non-canonical Wnt signaling has been shown to suppress adipogenesis, which, in turn, promoted the differentiation of mesenchymal stem cells into osteoblast lineage cells (65).
With the development of stem cell therapy, several scholars have hypothesized that stem cells may obtain improved regeneration ability in the inflammatory microenvironment (66). When they interact with the inflammatory microenvironment, stem cells can repair themselves and secret more regulatory factors to affect surrounding cells to promote regeneration. In turn, surrounding cells, such as tissue-resident immune cells, can influence the fate and behavior of stem cells by releasing immune modulators, and further contribute to tissue regeneration. Recently, it was observed that Wnt5a was abundantly expressed in the regenerating gastric tissue following Helicobacter pylori infection (67). Conditional deletion of Wnt5a or depletion of innate lymphoid cells, the cells that act as the main source of Wnt5a, reduced Mist1 ${ }^{+}$stem cell proliferation and impaired healing and regeneration in experimental gastric ulcer (67). This in vivo study suggested that Wnt5a may contribute to the interaction between stem cells and immune cells under inflammatory microenvironment. The effect of Wnt5a on periodontal regeneration under the inflammatory microenvironment is at the initial stages of being explored. An in vitro study demonstrated that the Wnt5a-mediated $\mathrm{Ca}^{2+}$ signaling pathway had an inhibitory role in cementogenic/osteogenic differentiation of HPDLCs following stimulation by pro-inflammatory cytokines IL-6 and TNF- $\alpha$ (68). However, another study suggested that the inflammatory microenvironment may suppress the $\mathrm{Wnt} / \mathrm{Ca}^{2+}$ signaling pathway and lead to reduced osteogenic differentiation of HPDLCs (69). This discrepancy may be due partly to the pleiotropic effects of Wnt5a in periodontal regeneration in inflammatory microenvironments. Wnt5a not only influences the regeneration potential of stem cells, but also has an impact on immune cells. Further studies in vivo will be required to fully understand the effect of Wnt5a on periodontal regeneration in a synthetic in vivo environment.

In addition, periodontal regeneration emphasizes the reconstruction of integrated periodontal tissues, not just the regeneration of hard tissues. Regeneration of periodontal ligament guarantees functional recovery and long-term stability of periodontal tissues. At present, and although further research is warranted, Wnt5a is hypothesized to be a candidate molecule for periodontal regeneration due to its ability to promote fibrillogenesis and to moderate osteogenesis in periodontal stem/progenitor cells.

\section{Conclusion}

As a prototypical non-canonical Wnt, Wnt5a is involved in many processes of periodontal tissue development, maintenance, and periodontitis. Based on its broad biological functions, Wnt5a may also serve a role in periodontal regeneration. However, current studies have primarily conducted in vitro experiments, so there is a lack of in vivo studies to verify the results. Moreover, the specific mechanism underlying Wnt5a regulation of periodontal homeostasis is not completely understood. Therefore, identifying how to regulate Wnt5a to maintain the balance between osteogenesis and osteoclastosis in periodontal tissues is important. Moreover, Wnt5a might display a diagnostic value in periodontitis as Wnt5a can be detected in GCF, and its expression level is correlated with 
the diseased site. Future studies should investigate whether the protein expression levels of Wnt5a in the GCF of patients with chronic periodontitis are related to the active and stable phases of the diseased site, as well as assessing the predictive value of Wnt5a.

\section{Acknowledgements}

Not applicable.

\section{Funding}

The present study was supported by the National Natural and Science Foundation of China (grant no. 81771077) and the Key Research and Development Program of Sichuan Province (grant no. 2020YFS0175).

\section{Availability of data and materials}

Not applicable.

\section{Authors' contributions}

XW and QL performed the literature search, wrote the manuscript and designed the figures. SG and YW designed the framework of this review and modified the manuscript. All authors read and approved the final manuscript.

\section{Ethics approval and consent to participate}

Not applicable.

\section{Patient consent for publication}

Not applicable.

\section{Competing interests}

The authors declare that they have no competing interests.

\section{References}

1. Cho MI and Garant PR: Development and general structure of the periodontium. Periodontology 2000 24: 9-27, 2000.

2. Luan X, Zhou X, Trombetta-eSilva J, Francis M, Gaharwar AK, Atsawasuwan P and Diekwisch TGH: MicroRNAs and periodontal homeostasis. J Dent Res 96: 491-500, 2017.

3. Mombelli A: Microbial colonization of the periodontal pocket and its significance for periodontal therapy.Periodontology 2000 76: 85-96, 2018.

4. Wang J, Massoudi D, Ren Y, Muir AM, Harris SE, Greenspan DS and Feng JQ: BMP1 and TLL1 are required for maintaining periodontal homeostasis. J Dent Res 96: 578-585, 2017.

5. Fujita A, Morimatsu M, Nishiyama M, Naruse K and Takashiba S: Mechanical stress modulates the homeostasis of periodontal ligament. Mol Biol Cell 29: 1, 2018.

6. Li J, Ke X, Yan F, Lei L and Li H: Necroptosis in the periodontal homeostasis: Signals emanating from dying cells. Oral Dis 24: 900-907, 2018.

7. Jin Y, Liu DX and Lin XP: IL-35 may maintain homeostasis of the immune microenvironment in periodontitis. Exp Ther Med 14: 5605-5610, 2017.

8. Xie X, Wang J, Wang K, Li C, Zhang S, Jing D, Xu C, Wang X, Zhao $\mathrm{H}$ and Feng JQ: Axin $2^{+}$-mesenchymal PDL cells, instead of $\mathrm{K} 14^{+}$epithelial cells, play a key role in rapid cementum growth. J Dent Res 98: 1262-1270, 2019.
9. Gavin BJ, McMahon JA and McMahon AP: Expression of multiple novel Wnt-1/int-1-related genes during fetal and adult mouse development. Genes Dev 4: 2319-2332, 1990.

10. Clark CC, Cohen I, Eichstetter I, Cannizzaro LA, McPherson JD, Wasmuth JJ and Iozzo RV: Molecular cloning of the human proto-oncogene Wnt-5A and mapping of the gene (WNT5A) to chromosome 3p14-p21. Genomics 18: 249-260, 1993.

11. Bauer M, Bénard J, Gaasterland T, Willert K and Cappellen D: WNT5A encodes two isoforms with distinct functions in cancers. PLoS One 8: e80526, 2013.

12. Kumawat K and Gosens R: WNT-5A: Signaling and functions in health and disease. Cell Mol Life Sci 73: 567-587, 2016.

13. Butler MT and Wallingford JB: Planar cell polarity in development and disease. Nat Rev Mol Cell Biol 18: 375-388, 2017.

14. De A: Wnt $/ \mathrm{Ca}^{2+}$ signaling pathway: A brief overview. Acta Biochim Biophys Sin (Shanghai) 43: 745-756, 2011.

15. Niehrs $\mathrm{C}$ and Acebron SP: Mitotic and mitogenic Wnt signalling. EMBO J 31: 2705-2713, 2012.

16. Wang D, Zhang Y and Shen C: Research update on the association between SFRP5, an anti-inflammatory adipokine, with obesity, type 2 diabetes mellitus and coronary heart disease. J Cell Mol Med 24: 2730-2735, 2020.

17. Yamaguchi TP, Bradley A, McMahon AP and Jones S: A Wnt5a pathway underlies outgrowth of multiple structures in the vertebrate embryo. Development 126: 1211-1223, 1999.

18. Bisson JA, Mills B, Paul Helt JC, Zwaka TP and Cohen ED: Wnt5a and Wnt11 inhibit the canonical Wnt pathway and promote cardiac progenitor development via the caspase-dependent degradation of AKT. Dev Biol 398: 80-96, 2015.

19. Li CG, Xiao J, Hormi K, Borok Z and Minoo P: Wnt5a participates in distal lung morphogenesis. Dev Biol 248: 68-81, 2002.

20. Pashirzad M, Shafiee M, Rahmani F, Behnam-Rassouli R, Hoseinkhani F, Ryzhikov M, Moradi Binabaj M, Parizadeh MR, Avan A and Hassanian SM: Role of Wnt5a in the pathogenesis of inflammatory diseases. J Cell Physiol 232: 1611-1616, 2017.

21. Asem MS, Buechler S, Wates RB, Miller DL and Stack MS: Wnt5a signaling in cancer. Cancers (Basel) 8: 79, 2016.

22. Lin M, Li L, Liu C, Liu H, He F, Yan F, Zhang Y and Chen Y: Wnt5a regulates growth, patterning, and odontoblast differentiation of developing mouse tooth. Dev Dyn 240: 432-440, 2011.

23. Cai J, Mutoh N, Shin JO, Tani-Ishii N, Ohshima H, Cho SW and Jung HS: Wnt5a plays a crucial role in determining tooth size during murine tooth development. Cell Tissue Res 345: 367-377, 2011.

24. Sarkar L and Sharpe PT: Expression of Wnt signalling pathway genes during tooth development. Mech Dev 85: 197-200, 1999.

25. Peng L, Ren LB, Dong G, Wang CL, Xu P, Ye L and Zhou XD: Wnt5a promotes differentiation of human dental papilla cells. Int Endod J 43: 404-412, 2010.

26. Xiang L, Chen M, He L, Cai B, Du Y, Zhang X, Zhou C, Wang C, Mao JJ and Ling J: Wnt5a regulates dental follicle stem/progenitor cells of the periodontium. Stem Cell Res Ther 5: 135, 2014.

27. Hasegawa D, Wada N, Maeda H, Yoshida S, Mitarai H, Tomokiyo A, Monnouchi S, Hamano S, Yuda A and Akamine A: Wnt5a induces collagen production by human periodontal ligament cells through TGF $\beta 1$-mediated upregulation of periostin expression. J Cell Physiol 230: 2647-2660, 2015.

28. Fu HD, Wang BK, Wan ZQ, Lin H, Chang ML and Han GL: Wnt5a mediated canonical Wnt signaling pathway activation in orthodontic tooth movement: Possible role in the tension force-induced bone formation. J Mol Histol 47: 455-466, 2016.

29. Wu XS, Hu L, Li Y, Wang F, Ma P, Wang J, Zhang C, Jiang C and Wang S: SCAPs regulate differentiation of DFSCs during tooth root development in swine. Int J Med Sci 15: 291-299, 2018.

30. Sakisaka $Y$, Tsuchiya M, Nakamura T, Tamura M, Shimauchi $H$ and Nemoto E: Wnt5a attenuates Wnt3a-induced alkaline phosphatase expression in dental follicle cells. Exp Cell Res 336: 85-93, 2015.

31. Wise G: Cellular and molecular basis of tooth eruption. Orthod Craniofac Res 12: 67-73, 2009.

32. Gopinathan G, Foyle D, Luan X and Diekwisch TGH: The Wnt antagonist SFRP1: A key regulator of periodontal mineral homeostasis. Stem Cells Dev 28: 1004-1014, 2019.

33. Rios HF, Ma D, Xie Y, Giannobile WV, Bonewald LF, Conway SJ and Feng JQ: Periostin is essential for the integrity and function of the periodontal ligament during occlusal loading in mice. J Periodontol 79: 1480-1490, 2008.

34. Panchamanon P, Pavasant $P$ and Leethanakul C: Periostin plays role in force-induced stem cell potential by periodontal ligament stem cells. Cell Biol Int 43: 506-515, 2019. 
35. Kobayashi Y, Thirukonda GJ, Nakamura Y, Koide M Yamashita T, Uehara S, Kato H, Udagawa $\mathrm{N}$ and Takahashi $\mathrm{N}$ : Wnt16 regulates osteoclast differentiation in conjunction with Wnt5a. Biochem Biophys Res Commun 463: 1278-1283, 2015.

36. Maeda K, Kobayashi Y, Udagawa N, Uehara S, Ishihara A, Mizoguchi T, Kikuchi Y, Takada I, Kato S, Kani S, et al: Wnt5a-Ror2 signaling between osteoblast-lineage cells and osteoclast precursors enhances osteoclastogenesis. Nat Med 18: 405-412, 2012

37. Roberts JL, Liu G, Paglia DN, Kinter CW, Fernandes LM, Lorenzo J, Hansen MF, Arif A and Drissi H: Deletion of Wnt5a in osteoclasts results in bone loss through decreased bone formation. Ann N Y Acad Sci 1463: 45-59, 2020

38. Divaris K, Monda KL, North KE, Olshan AF, Reynolds LM, Hsueh WC, Lange EM, Moss K, Barros SP, Weyant RJ, et al: Exploring the genetic basis of chronic periodontitis: A genomewide association study. Hum Mol Genet 22: 2312-2324, 2013.

39. Nanbara H, Wara-aswapati N, Nagasawa T, Yoshida Y, Yashiro R, Bando Y, Kobayashi H, Khongcharoensuk J, Hormdee D, Pitiphat $\mathrm{W}$, et al: Modulation of Wnt5a expression by periodontopathic bacteria. PLoS One 7: e34434, 2012

40. Maekawa T, Kulwattanaporn P, Hosur K, Domon H, Oda M, Terao Y, Maeda T and Hajishengallis G: Differential expression and roles of secreted frizzled-related protein 5 and the wingless homolog Wnt5a in periodontitis. J Dent Res 96: 571-577, 2017.

41. Haftcheshmeh SM, Mohammadi A, Soltani A, MomtaziBorojeni AA and Sattari M: Evaluation of STAT1 and Wnt5a gene expression in gingival tissues of patients with periodontal disease. J Cell Biochem 120: 1827-1834, 2019.

42. Chatzopoulos GS, Mansky KC, Lunos S, Costalonga M and Wolff LF: Sclerostin and WNT-5a gingival protein levels in chronic periodontitis and health. J Periodont Res 54: 555-565, 2019.

43. Ge XP, Can YH, Zhang CG, Zhou CY, Ma KT, Meng JH and Ma XC: Requirement of the NF- $\kappa \mathrm{B}$ pathway for induction of Wnt-5A by interleukin-1 $\beta$ in condylar chondrocytes of the temporomandibular joint: Functional crosstalk between the Wnt-5A and $\mathrm{NF}-\kappa \mathrm{B}$ signaling pathways. Osteoarthritis Cartilage 19: 111-117, 2011

44. Naskar D, Maiti G, Chakraborty A, Roy A, Chattopadhyay D and Sen M: Wnt5a-Rac1-NF-кB homeostatic circuitry sustains innate immune functions in macrophages. J Immunol 192: 4386-4397, 2014

45. Zhang Q, Liu J, Ma L, Bai $\mathrm{N}$ and $\mathrm{Xu} \mathrm{H}$ : Wnt5a is involved in LOX-1 and TLR4 induced host inflammatory response in peri-implantitis. J Periodont Res 55: 199-208, 2020.

46. Pereira C, Schaer DJ, Bachli EB, Kurrer MO and Schoedon G: Wnt5A/CaMKII signaling contributes to the inflammatory response of macrophages and is a target for the antiinflammatory action of activated protein $\mathrm{C}$ and interleukin-10. Arterioscler Thromb Vasc Biol 28: 504-510, 2008.

47. Ouchi N, Higuchi A, Ohashi K, Oshima Y, Gokce N, Shibata R, Akasaki Y, Shimono A and Walsh K: Sfrp5 is an antiinflammatory adipokine that modulates metabolic dysfunction in obesity. Science 329: 454-457, 2010.

48. Schulz J, Knappe C, Graetz C, Mewes L, Türk K, Black AK, Lieb W, Schäfer AS, Fawzy El-Sayed KM, Dörfer CE, et al: Secreted frizzled-related protein 5 serum levels in human periodontitis-A nested case-control study. J Clin Periodontol 46: 522-528, 2019

49. Schulte DM, Müller N, Neumann K, Oberhäuser F, Faust M, Güdelhöfer H, Brandt B, Krone W and Laudes M: Pro-inflammatory wnt5a and anti-inflammatory sFRP5 are differentially regulated by nutritional factors in obese human subjects. PLoS One 7: e32437, 2012

50. Tong S, Du Y, Ji Q, Dong R, Cao J, Wang Z, Li W, Zeng M, Chen H, Zhu X and Zhou Y: Expression of Sfrp5/Wnt5a in human epicardial adipose tissue and their relationship with coronary artery disease. Life Sci 245: 117338, 2020.

51. Lu YC, Wang CP, Hsu CC, Chiu CA, Yu TH, Hung WC, Lu LF Chung FM, Tsai IT, Lin HC and Lee YJ: Circulating secreted frizzled-related protein 5 (Sfrp5) and wingless-type MMTV integration site family member 5a (Wnt5a) levels in patients with type 2 diabetes mellitus. Diabetes Metab Res Rev 29: 551-556, 2013.

52. Cho YK, Kang YM, Lee SE, Lee Y, Seol SM, Lee WJ, Park JY and Jung CH: Effect of SFRP5 (secreted frizzled-related protein 5) on the WNT5A (wingless-type family member 5A)-induced endothelial dysfunction and its relevance with arterial stiffness in human subjects. Arterioscler Thromb Vasc Biol 38: 1358-1367, 2018.
53. Mehmeti M, Bergenfelz C, Kallberg E, Millrud CR, Björk P, Ivars F, Johansson-Lindbom B, Kjellström S, André I and Leandersson K: Wnt5a is a TLR2/4-ligand that induces tolerance in human myeloid cells. Commun Biol 2: 176, 2019.

54. Feng Y, Liang Y, Zhu X, Wang M, Gui Y, Lu Q, Gu M, Xue X, Sun $\mathrm{X}, \mathrm{He} \mathrm{W}$, et al: The signaling protein Wnt5a promotes TGFß1-mediated macrophage polarization and kidney fibrosis by inducing the transcriptional regulators Yap/Taz. J Biol Chem 293: 19290-19302, 2018

55. Gao YC, Wen Q, Hu SF, Zhou X, Xiong W, Du X, Zhang L,

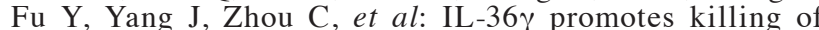
Mycobacterium tuberculosis by macrophages viaWNT5A-induced noncanonical WNT signaling. J Immunol 203: 922-935, 2019.

56. Chen FM and Jin Y: Periodontal tissue engineering and regeneration: Current approaches and expanding opportunities. Tissue Eng Part B Rev 16: 219-255, 2010.

57. Liu N, Gu B, Liu N, Nie X, Zhang B, Zhou X and Deng M: Wnt/ $\beta$-catenin pathway regulates cementogenic differentiation of adipose tissue-deprived stem cells in dental follicle cellconditioned medium. PLoS One 9: e93364, 2014

58. Zhang F, Luo K, Rong Z, Wang Z, Luo F, Zhang Z, Sun D, Dong S, $\mathrm{Xu} \mathrm{J}$ and Dai F: Periostin upregulates Wnt/ $\beta$-catenin signaling to promote the osteogenesis of CTLA4-modified human bone marrow-mesenchymal stem cells. Sci Rep 7: 41634, 2017.

59. Morsczeck C, Reck A and Reichert TE: WNT5A supports viability of senescent human dental follicle cells. Mol Cell Biochem 455: 21-28, 2019.

60. Yang X, Zhang C, Jiang J and Li Y: Baicalein retards proliferation and collagen deposition by activating p38MAPK-JNK via microRNA-29. J Cell Biochem 120: 15625-15634, 2019.

61. Zhao Y, Wang H, Li X, Cao M, Lu H, Meng Q, Pang H, Li H, Nadolny C, Dong X and Cai L: Ang II-AT1R increases cell migration through PI3K/AKT and NF- $\mathrm{KB}$ pathways in breast cancer. J Cell Physiol 229: 1855-1862, 2014.

62. Hasegawa D, Wada N, Yoshida S, Mitarai H, Arima M, Tomokiyo A, Hamano S, Sugii H and Maeda H: Wnt5a suppresses osteoblastic differentiation of human periodontal ligament stem cell-like cells via Ror2/JNK signaling. J Cell Physiol 233: 1752-1762, 2018

63. Nemoto E, Sakisaka Y, Tsuchiya M, Tamura M, Nakamura T, Kanaya S, Shimonishi M and Shimauchi H: Wnt3a signaling induces murine dental follicle cells to differentiate into cementoblastic/osteoblastic cells via an osterix-dependent pathway. J Periodont Res 51: 164-174, 2016.

64. Nemoto E, Ebe Y, Kanaya S, Tsuchiya M, Nakamura T, Tamura M and Shimauchi H: Wnt5a signaling is a substantial constituent in bone morphogenetic protein-2-mediated osteoblastogenesis. Biochem Biophys Res Commun 422: 627-632, 2012.

65. Hashimoto Y, Matsuzaki E, Higashi K, Takahashi-Yanaga F, Takano A, Hirata M and Nishimura F: Sphingosine-1-phosphate inhibits differentiation of C3H10T1/2 cells into adipocyte. Mol Cell Biochem 401: 39-47, 2015.

66. Fawzy El-Sayed KM, Elahmady M, Adawi Z, Aboushadi N, Elnaggar A, Eid M, Hamdy N, Sanaa D and Dörfer CE: The periodontal stem/progenitor cell inflammatory-regenerative cross talk: A new perspective. J Periodont Res 54: 81-94, 2019.

67. Nienhuser H, Kim W, Malagola E, Ruan T, Valenti G, Middelhoff M, Bass A, Der CJ, Hayakawa Y and Wang TC: Mist1 ${ }^{+}$ gastric isthmus stem cells are regulated by Wnt5a and expand in response to injury and inflammation in mice. Gut: Jul 24, 2020 (Epub ahead of print). doi: 10.1136/gutjnl-2020-320742.

68. Han P, Lloyd T, Chen Z and Xiao Y: Proinflammatory cytokines regulate cementogenic differentiation of periodontal ligament cells by $\mathrm{Wnt} / \mathrm{Ca}(2+)$ signaling pathway. J Interferon Cytokine Res 36: 328-337, 2016

69. Liu N, Shi S, Deng M, Tang L, Zhang G, Liu N, Ding B, Liu W, Liu Y, Shi H, et al: High levels of $\beta$-catenin signaling reduce osteogenic differentiation of stem cells in inflammatory microenvironments through inhibition of the noncanonical Wnt pathway. J Bone Miner Res 26: 2082-2095, 2011.

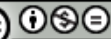

This work is licensed under a Creative Commons Attribution-NonCommercial-NoDerivatives 4.0 International (CC BY-NC-ND 4.0) License. 